\title{
Validation of Microchip RT-PCR COVID-19 Detection System
}

\author{
Kelsey de Campos-Stairiker ${ }^{1}$, Asha Shravanthi Pidathala ${ }^{1}$, Rajwant K. Gill², Irina Gelimson², \\ Natallia Varankovich², Sikander S. Gill2*, Maxim Slyadnev², Sonia Kapur'1 \\ ${ }^{1}$ Ayumetrix, Lake Oswego, OR, USA \\ ${ }^{2}$ Lumex Instruments Canada, Mission, BC, Canada \\ Email: *sikander.gill@lumexinstruments.com
}

How to cite this paper: de Campos-Stairiker, K., Pidathala, A.S., Gill, R.K., Gelimson, I., Varankovich, N., Gill, S.S., Slyadnev, M. and Kapur, S. (2021) Validation of Microchip RT-PCR COVID-19 Detection System. Journal of Biosciences and Medicines, 9, 8-24. https://doi.org/10.4236/jbm.2021.99002

Received: July 31, 2021

Accepted: August 31, 2021

Published: September 3, 2021

Copyright $\odot 2021$ by author(s) and Scientific Research Publishing Inc. This work is licensed under the Creative Commons Attribution International License (CC BY 4.0).

http://creativecommons.org/licenses/by/4.0/

\begin{abstract}
While meeting the pandemic demand of SARS-CoV-2 testing, clinical laboratories worldwide tend to adopt new test systems offering cost-effective and faster test outcomes. However, the reliability of SARS-CoV-2 test results has paramount importance in the management of such a health crisis. Therefore, this study sought to determine the accuracy of the test results from a novel duplex Microchip RT-PCR test system using patient saliva samples and nasal swabs stabilized in Viral Transport Medium (VTM) with reference threshold Cycle Values (Ct). The VTM used to stabilize these samples during transport was found to be inhibitory to the RT-PCR. Therefore, all the samples were subjected to spin column purification of total RNA to remove the influence of VTM. A total of 70 patient samples, including 24 positive- and 31 negative-saliva in VTM samples and 15 positive nasal swab samples, were tested. Results obtained from both the sample types were compared to their reference values and no false positive or false negatives were observed. From this data, accuracy, specificity, and sensitivity were determined to be $100 \%$ applying the corresponding formulae. The limit of detection with $95 \%$ confidence probability was determined to be 2.5 copies $/ \mu$ in the original sample.
\end{abstract}

\section{Keywords}

SARS-CoV-2, Microchip, RT-PCR, Duplex, Nasal, Saliva, LoD

\section{Introduction}

Widespread and rapid testing of the newly emerged pandemic Coronavirus Disease 2019 (COVID-19) is needed to curb the spread of the causative virus SARSCoV-2. The globally preferred method for diagnosis is one-step real-time Reverse Transcriptase PCR (RT-PCR) [1] [2] [3] [4]. However, the pandemic supply 
shortages, and widespread implementation of testing has severely limited the access to the test kits. To minimize the volume needed for PCR reagents, thus allowing the reagent stock to last longer, a miniaturized disposable microchip-based real-time RT-PCR test system has emerged for the detection of SARS-CoV-2 [5] [6].

In this novel technology, a cost-effective TaqMan chemistry reaction of $1.2 \mu \mathrm{l}$ is accommodated in each of the microwells of the 30- or 48 microwell formats of the microchip. The 1-step RT-PCR reaction in the microwells involves the US $\mathrm{CDC}$ recommended primers and probes

[https://www.idtdna.com/pages/landing/coronavirus-research-reagents/cdc-assays]. The N1 and N2 primers and probe detect the nucleocapsid (N) gene of SARS$\mathrm{CoV}-2$ while the Hs primers and probe detect the HsRPP30 gene in human specimen control. Microchips with 30-microwell format in singleplex offer a throughput of 7 unknown samples in addition to the controls [5]. In other 30- and 48-microwell formats, the $\mathrm{N} 1$ molecular target is paired with $\mathrm{Hs}$ as a duplex offering a throughput of 27 or 45 samples in addition to the controls in respective microchip formats [6]. The primers and probes of these molecular targets are preloaded and lyophilized in the individual microwells of the microchips, and 45 cycles of 1-step RT-PCR are performed in 32 minutes [5] [6].

The microchip consists of an aluminum metal plate with stamped microwells which provides high heat transfer efficiency and offers a high surface area to volume ratio with a $1.2 \mu \mathrm{l}$ reaction well [5]. These attributes of product design combine to give high heating and cooling rates, low reagent consumption, rapid results, and are overall cost-effective [7]-[12]. These attributes also allow this system to be a candidate to become an effective workhorse in the current or post-pandemic scenario.

For the majority of the RT-PCR methods, RNA extraction from the test samples is normally required [13] [14]. However, the supply chains of the extraction kits are also hit by the pandemic and this scarcity of the extraction kits warrants the need for validation of new RNA extraction kits or the development of extraction-free RT-PCR testing to ease supply constraints. Although the RNA extraction kits and procedures add to the cost of the test and drain on the test lab resources, it is essential to process sample specimens stabilized and transported in Viral Transport Medium (VTM) that may have an inhibitory impact on RT-PCR [15] [16] [17]. The Microchip RT-PCR Covid-19 Test System was validated with nasal swabs and saliva samples in VTM (saliva-VTM samples) using spin columnbased RNA extraction, determining analytical and diagnostic specificity, amplification efficiency, and reproducibility of results.

\section{Materials \& Methods}

Throughout the laboratory and manufacturing preparations and procedures, good laboratory practices (GLP) and good manufacturing practices (GMP) were followed

[https://www.canada.ca/en/health-canada/services/drugs-health-products/natura 
l-non-prescription/legislation-guidelines/guidance-documents/good-manufactu ring-practices.html].

Reagents: The following reagents were procured, prepared and used in these studies:

1) RT-PCR reagents: The RT-PCR reagents included the followings:

a) Primers and probes of test kits: The primers-probes included in the 2019-nCoV CDC EUA Kit were obtained from Integrated DNA Technology Inc, USA (Cat \#10006770, https://www.idtdna.com) to detect N1 and N2 region of the $\mathrm{N}$ gene of SARS-CoV-2 virus, and to detect HsRPP30, a house keeping gene of human sample as an Internal Control (IC).

b) 1-Step RT PCR Master mix: Ultra Plex ${ }^{\mathrm{TM}}$ 1-Step ToughMix ${ }^{\circledR}$, a $4 \mathrm{X}$ concentrated master mix for 1-step qRT-PCR containing dATP, dCTP, dGTP, dTTP, magnesium, qScript XLT reverse transcriptase, RNase inhibitor protein and AccuStart II hot-start Taq DNA polymerase (Catalogue \#95166-01K, from Quanta Bio, USA, https://www.quantabio.com) was utilized as the TaqMan Taq polymerase enzyme premix for real-time PCR on the microchip.

c) RT-PCR controls: As a positive control in all experiments, genomic RNA extracted from heat-inactivated SARS-related Coronavirus 2, isolate USA-WA1/2020 that includes the viral sequences for the N1 and N2 molecular targets in the RNA of SARS-CoV-2, $5.0 \times 10^{7}$ genome equivalents/ml (Catalog \#NR-52347, BEI Resources, USA, https://www.beiresources.org) was used.

2) Preparation of viral RNA: Preparation of 10 -fold dilution series $\left(5 \times 10^{4}, 5\right.$ $\times 10^{3}, 5 \times 10^{2}, 5 \times 10^{1}$, and $5 \times 10^{0}$ copies/ $\mu$ l) of the SARS-CoV-2 viral RNA was carried with RNA storage solution cat \# AM7001 purchased from Thermo Fisher Scientific, Canada (https://www.thermofisher.com). Similarly, a 2-fold serial dilution $(1000,500,250,125,62.5,31.3$, and 15.6 copies/ $\mu l)$ was generated for the viral RNA. Each dilution was tested by the one-step duplex RT-PCR assay using $1 \mu \mathrm{l}$ in RT-PCR mix following the Instructions for use supplied by Lumex Instruments, Canada, https://www.lumexinstruments.com.

3) Sample types and processing: The following types of samples were obtained on site or through shipments and stored at $4^{\circ} \mathrm{C}$ in refrigerator or $-20^{\circ} \mathrm{C}$ or $-80^{\circ} \mathrm{C}$ for long-term storage.

a) Nasal swab samples: 15 de-identified nasal swab positive samples suspended in about $3 \mathrm{ml}$ of Viral Transport Medium (VTM) were obtained from CLIA certified reference lab.

i) The experiment to characterize the supernate and swab consisted of 3 samples chosen with low, mid, and high reference $\mathrm{Ct}$ values. Un-squeezed swabs were taken out from the original tube after vortexing for $2 \mathrm{~min}$, and then the swab was suspended and washed in $200 \mu \mathrm{l}$ RNA storage buffer followed by transferring 100 $\mu \mathrm{l}$ of this solution into the spin column for extraction. The volume of the soaked solution remaining in the swab was approximated to be about $25 \mu$ l.

ii) If not otherwise stated, in subsequent experiments only supernate was used in spin column purification. 
b) Saliva-VTM samples: 24 de-identified positive saliva samples with reference $\mathrm{Ct}$ values and 31 reference negative samples were obtained mixed as 1:1 ratio with the VTM as a total suspension of $3 \mathrm{ml}$ in polypropylene tubes from CLIA certified reference lab and referred as saliva-VTM samples in this presentation.

c) RNA extraction: Viral RNA was extracted manually from clinical or general nasal samples and saliva-VTM samples using Total RNA Purification Kit from Norgen Biotek Corp, Canada (Cat. 17200, https://www.norgenbiotek.com). The purification is based on spin column chromatography using micro centrifuge for spinning the columns. The extracted RNA was frozen and stored at $-80^{\circ} \mathrm{C}$. For spin column purification, the manufacturer's protocol was followed with the following modification: the modified step involved mixing of $100 \mu \mathrm{l}$ of the sample in prescribed volume of the lysis buffer.

4) Preparation of disposable pre-filled microchips: The empty microchips were manufactured with 30 microwells ( 6 columns $\times 5$ rows) by metal stamping technology and coated with surface modifiers [5] [6]. The empty microchips were eventually filled with $1.2 \mu \mathrm{l}$ solution of primers and probes to manufacture both the formats deployed in the present studies: singleplex of 3 molecular targets $(\mathrm{N} 1, \mathrm{~N} 2$, and HS) and duplex of 2 molecular targets (N1/Hs) along with stabilizing agents using an OT2 robotic workstation from Opentrons, USA (https://www.opentrons.com) in a pre-defined layout (Figure 1(a), Figure 1(b)).

The prefilled microchips were subjected to lyophilization and then individually sealed with a desiccant in an air-tight package by Lumex Instruments, Canada (www.lumexinstruments.com/). A SJIA-10N Lyophilizer of Ningbo Shuangjia Instrument Co., Ltd, China (http://www.shuangjiayiqi.com/) was used for the process of lyophilization.

5) Microchip-based 1-step RT-PCR set up: The real-time RT-PCR assay was set up in the prefilled microchips and analysis was completed on the AriaDNA ${ }^{\mathrm{TM}}$ analyzer obtained from Lumex Instruments Canada, Mission, Canada (https://www.lumexinstruments.com) using manufacturer's software to control the instrument as follows:

a) RT-PCR set up in microchip: the RT-PCR mix of the sample and master mix was prepared in individual $200 \mu \mathrm{PCR}$ tubes maintained at $4^{\circ} \mathrm{C}$ in a cold block. The PCR mix was prepared as UltraPlex ${ }^{\mathrm{TM}} 1$-Step ToughMix ${ }^{\circledR}$ : sample RNA: nuclease free $\mathrm{H}_{2} \mathrm{O}$ in the volumetric ratio of 1:3:0. The Negative Template Control (NTC) was prepared in ratio of 1:0:3 by replacing the sample RNA with nuclease free $\mathrm{H}_{2} \mathrm{O}$. In the case of the Positive Template Control (PTC), the ratio of 1:1:2 was created by replacing the sample RNA by genomic RNA of SARS-Cov-2 $\left(1 \times 10^{4}\right.$ copies $)$.

Before loading the microwells with this reaction mix, $620 \mu \mathrm{l}$ of silicone oil was dispensed in the mid area of the microwell array. The PCR mix was quickly pipetted by submerged liquid handling under the oil layer. The objective of the overlaying oil layer is to contain the PCR mix in the microwells and to contain 


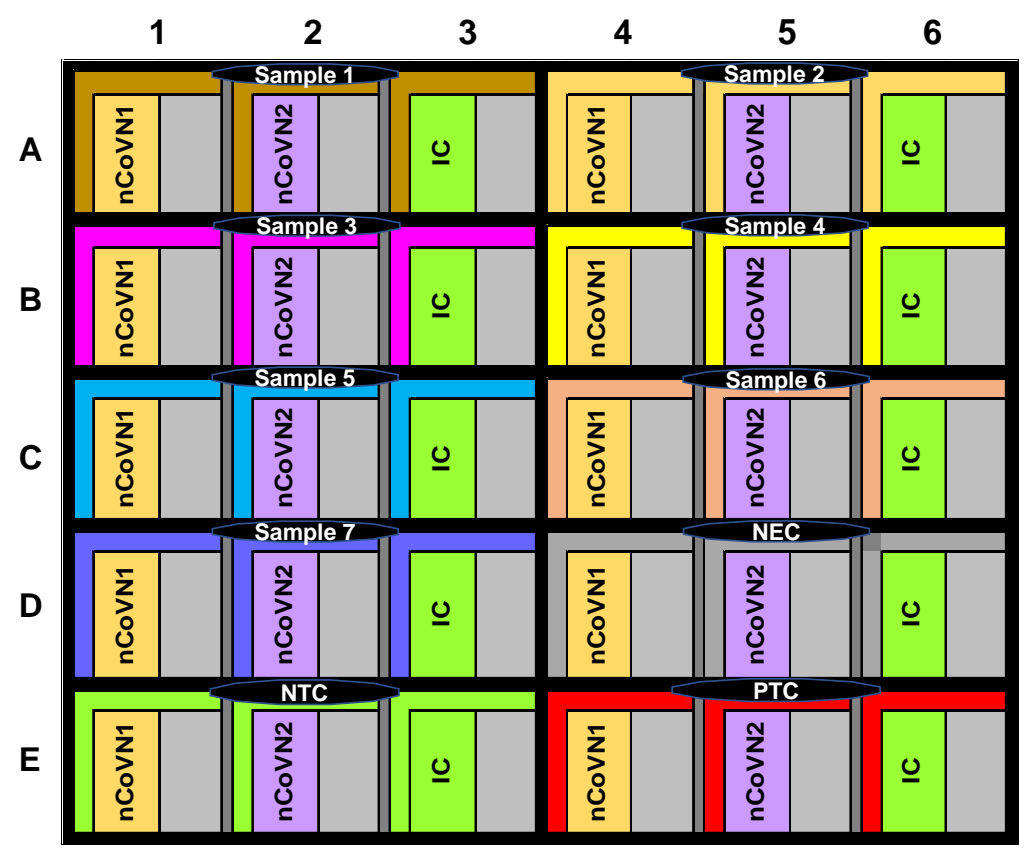

(a)

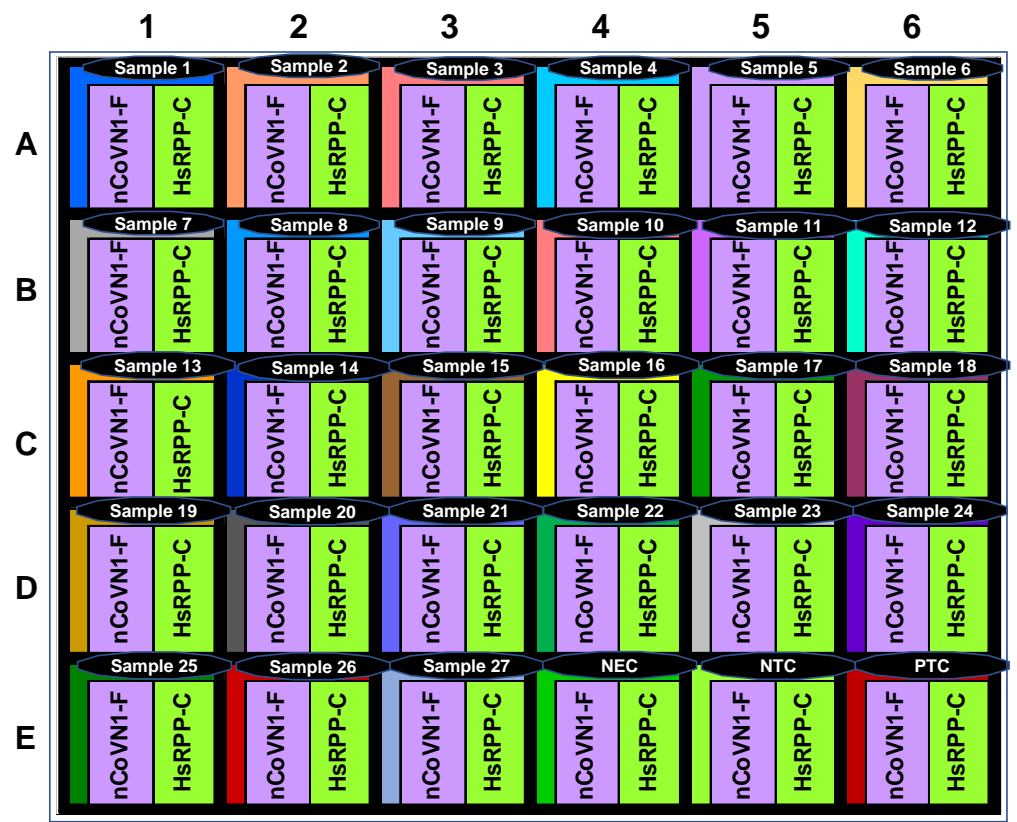

(b)

Figure 1. A 30-microwell formats: (a) singleplex, 3 molecular targets (N1, N2, and Hs). (b) duplex, 2 molecular targets (N1 and $\mathrm{Hs}$ ).

evaporation of the reagents during processing and thermocycling. The samples, NTC, NEC, and PTC are loaded into the microwells after inserting the microchip in the holder following the layout map of the microchips with $1.2 \mu \mathrm{l} /$ microwell. The RT-PCR was performed and analyzed after inserting the loaded microchips along with its holder into the AriaDNA ${ }^{\mathrm{TM}}$ PCR analyzer.

b) Thermocycling profile: Fast thermal settings were applied following Raz- 
van et al., 2021 [5], and Gill et al., 2021 [6] that include a reverse transcription step $\left(50^{\circ} \mathrm{C} \times 300 \mathrm{~s}\right)$, followed by a denaturing step $\left(95^{\circ} \mathrm{C} \times 120 \mathrm{~s}\right)$ and 45 cycles $\left(95^{\circ} \mathrm{C} \times 1 \mathrm{~s}\right)$ followed by extension and signal recording $\left(55^{\circ} \mathrm{C} \times 20 \mathrm{~s}\right)$.

6) PCR curve analysis: The Ct values were determined by pre-set second derivative maximum (SDM) and manually tweakable threshold setting once fluorescence has passed an auto-set SDM threshold [6]. The SDM value is reported when amplitude of the fluorescent signal and amplitude of its first derivative are both above the pre-set thresholds set at 150 and 30 arbitrary units, respectively.

In the Threshold mode, amplitude section of parameters in the Graph set up window of the software was adjusted so that the threshold values were set as $5 \%$ of the maximum intensity value of the respective detection channel. The threshold levels were adjusted from the threshold channel1 and threshold channel 2 fields independently for the respective detection channel.

7) Validation studies: The validation studies were performed as follows and the data was analyzed following Coetzee, 2004 [18]:

a) Accuracy: The accuracy of the reference samples on the test system, and thus the test outcome of the saliva-VTM and nasal swabs was determined using number of true positives (TP), true negatives (TN), and total number of samples as follows:

$$
\text { Accuracy }=(\mathrm{TP}+\mathrm{TN}) /(\text { Total number of samples })
$$

b) Specificity and sensitivity: The validation of specificity of duplex N1/Hs RT-PCR assay was evaluated by determining clinical performance of the duplex RT-PCR assay. It was evaluated by testing clinical specimens of upper respiratory tract nasal swabs in VTM and saliva-VTM samples of reference Ct values by calculating the number of TP, TN, False Positives (FP), and False Negatives (FN) among these samples. The specificity was calculated as follows:

$$
\mathrm{P}(\mathrm{T}-\mid \mathrm{D}-)=\mathrm{TN} /(\mathrm{TN}+\mathrm{FP})=\%
$$

where, $\mathrm{D}-=$ reference negative test; $\mathrm{T}-=$ negative test.

The proportion of patients with reference positive, who tested positive, conveys the sensitivity measure of the test system and it was calculated by the following formula:

$$
\mathrm{P}(\mathrm{T}+\mid \mathrm{D}+)=\mathrm{TP} /(\mathrm{TP}+\mathrm{FN})=\%
$$

where, $\mathrm{D}+=$ reference positive test; $\mathrm{T}+=$ positive test.

c) Determination of Limit of Detection (LoD): The analytical sensitivity in terms of LoD, defined as the lower limit above which an analyte is detected reliably. A 10-fold dilution series was used to determine approximate LoD. It was fine tuned using a 2-fold dilution series. The confirmation of the LoD was performed by testing 20 samples of extracted RNA. Thus, the LoD was confirmed by running 20-replicate experiments to attain approximately $95 \%$ of all (true positive) replicates test positive following CDC protocol.

(https://www.fda.gov/media/134922/download).

The number of RNA copies in reaction volume was calculated as follows: 
\#RNA copies per reaction [copies] =

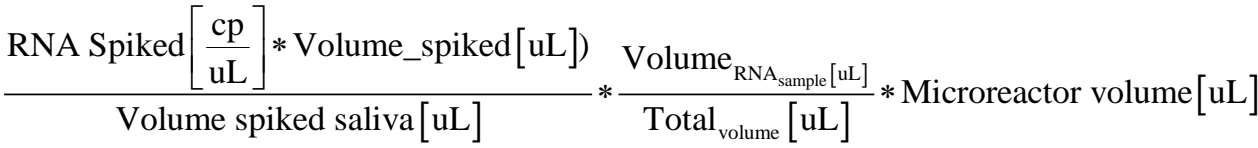

where,

RNA Spiked is the RNA concentration of the standard stock;

Volume Spiked is the volume of standard stock used in the assay;

Volume spiked saliva is the volume of negative saliva spiked with RNA;

Volume RNA sample is the aliquot of the Volume of negative saliva spiked with RNA to be used in the reaction Mix;

Total Volume is the resulting solution of RNA Sample, nuclease free water and master mix;

Microreactor volume is $1.2 \mu \mathrm{L}$ taken to perform RT-PCR in a microwell.

Statistical analysis: For analysis of data, basic statistical analysis, including mean, standard deviation, and percent coefficient of variation of the mean $\mathrm{Ct}$ value, were calculated.

\section{Results and Discussion}

Real-time RT-PCR is a powerful amplification tool that has become a standard for the detection of SARS-Cov-2 in the current pandemic [1] [2] [3] [4] [19]. Therefore, like any other diagnostic method, Microchip based RT-PCR for Covid-19 detection system was subjected to strict performance criteria to earn the reliability of the test results in the present studies. The process of validation of this detection system was started with singleplex microchip test system in which $\mathrm{N} 1$ and N2 molecular targets for SARS-CoV-2 detection and Hs for the detection of human specimen in the sample are FAM dye-based detection [6]. In addition, the duplex microchip test system also offered an opportunity to test performance criteria including analytical and diagnostic sensitivity, diagnostic specificity, and amplification efficiency.

Reproducibility of the results: Both the N1 and N2 SARS-CoV-2 targets along with the Hs target displayed highly reproducible amplification curves as shown in Table 1 and Figure 2 for $\mathrm{n}=9$ replicates: $\mathrm{Ct}(\mathrm{N} 1)$ of 26.17 with $\mathrm{CV}=$ $0.34 \%$; $\mathrm{Ct}(\mathrm{N} 2)$ of 26.07 with $\mathrm{CV}=0.81 \%$, and $\mathrm{Ct}(\mathrm{Hs})$ of 25.36 with $\mathrm{CV}=0.77 \%$ were obtained, respectively.

Extraction of RNA from samples: In preliminary experiments, the extraction-free methodology [20] was applied to three patient saliva-VTM samples: negative sample \#52, two positive samples \#79 and \#85 having reference Ct 19.1 and Ct 19.9, respectively. However, the microchip RT-PCR resulted in inhibited amplification of both the positive samples displaying significantly low intensity and delayed $\mathrm{Ct}$ for all the replicates $(\mathrm{n}=3)$ of each sample (data not shown). For comparison, two nuclease free water samples were processed through the extraction-free method after the addition of genomic RNA of SARS-CoV-2 $\left(1 \times 10^{4}\right.$ copies/ul) and were analyzed in the same microchip. All replicates of RNA 


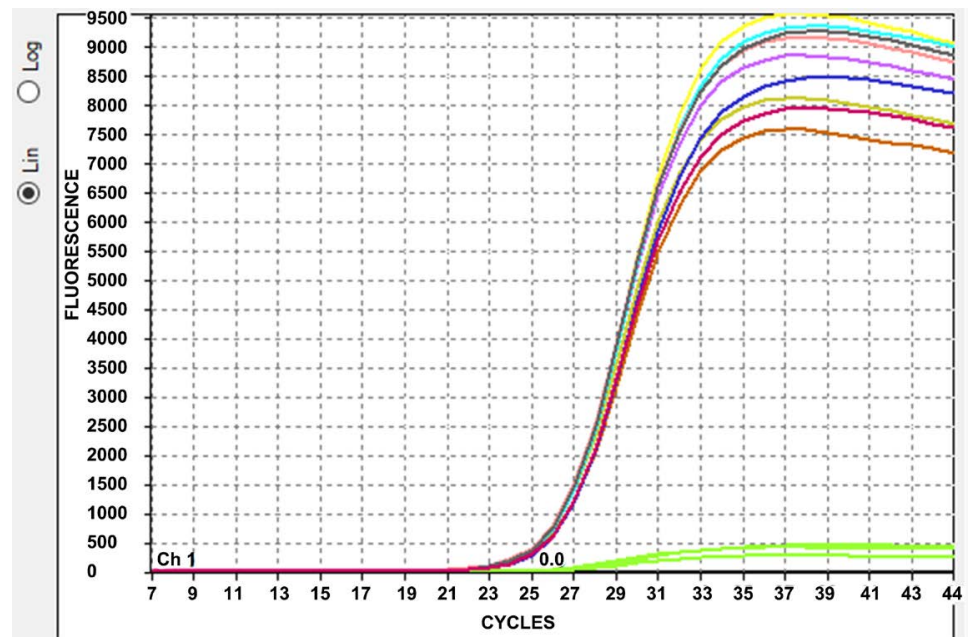

(a)

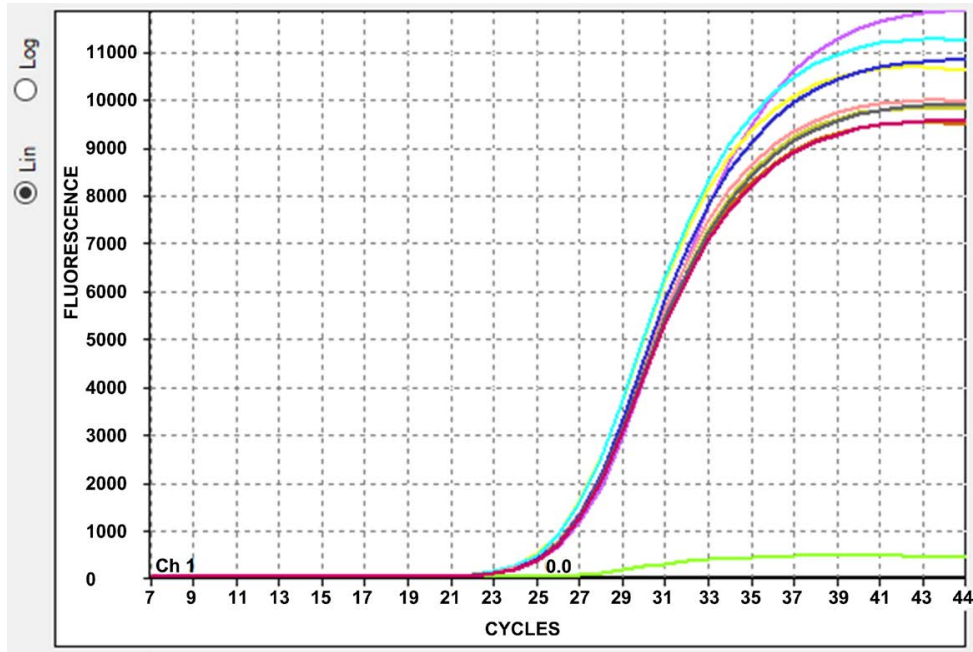

(b)

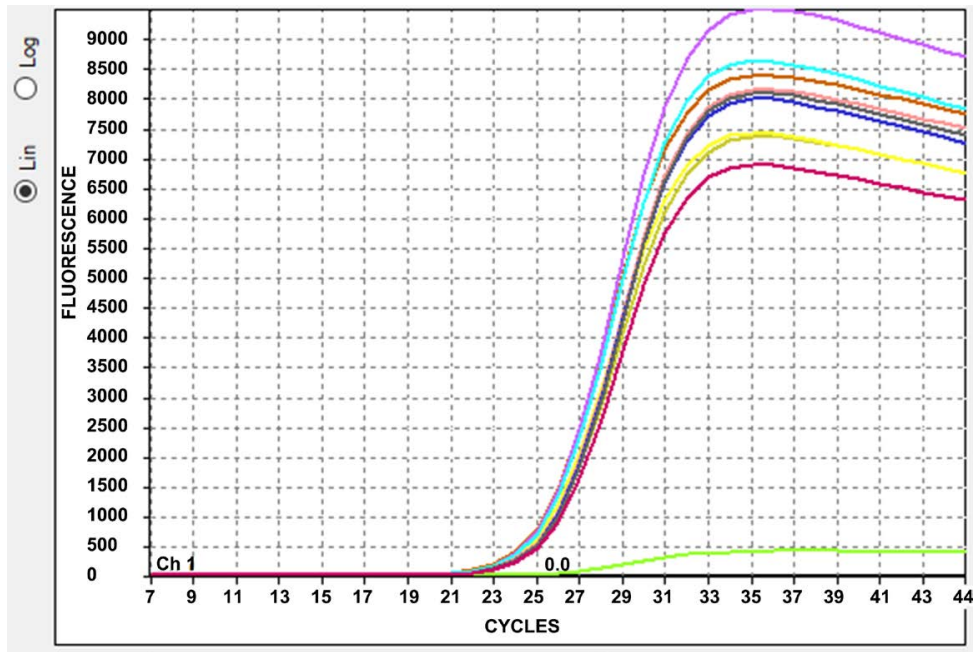

(c)

Figure 2. Reproducibility of amplification curves $(n=9)$ along with NTC (green curves) for molecular targets: (a) N1, (b) N2, and (c) Hs. The curves trailing close to the threshold are NTC. 
Table 1. Reproducibility of microchip RT-PCR test system.

\begin{tabular}{cccc}
\hline \multirow{2}{*}{ Replicates } & \multicolumn{2}{c}{ Microchip RT-PCR Ct } & IC Target \\
\cline { 2 - 4 } 1 & N1 & N2 & Hs \\
2 & 26.16 & 25.97 & 25.02 \\
3 & 26.11 & 26.03 & 25.42 \\
4 & 26.08 & 26.60 & 25.28 \\
5 & 26.29 & 25.87 & 25.19 \\
6 & 26.27 & 25.92 & 25.22 \\
7 & 26.10 & 26.02 & 25.52 \\
8 & 26.30 & 26.11 & 25.50 \\
9 & 26.09 & 26.03 & 25.60 \\
NTC & 26.15 & 26.05 & 25.53 \\
Mean & 0 & 0 & 0 \\
SD & 26.17 & 26.07 & 25.36 \\
CV\% & 0.09 & 0.21 & 0.20 \\
\hline
\end{tabular}

sample in water $(n=3)$ resulted in efficient amplification, indicating that the saliva-VTM samples suffered from inhibition by VTM, in accordance with observations of [16] [21].

In an attempt to remove the inhibition by VTM, a 2 -fold dilution series of the positive sample \#89 were analyzed. The 2 -fold dilution of the sample, resulted in Ct values of 20.31, 21.34, 23.16, 24.09, 25.23, and 26.45, respectively. The data suggested that dilution of sample 4- or 8-fold could potentially reduce the inhibition. However, to avoid dilution of the SARS-CoV-2 RNA which would eventually lead to a false negative result in low viral load samples [22] [23] [24], it would be desirable to process samples through an RNA purification step rather than diluting the sample.

To address this VTM inhibition, saliva-VTM samples were processed through purification procedure to become inhibitor-free. Since a spin column methodology is very user-friendly, this method was chosen to purify RNA from the clinical saliva-VTM samples. In preliminary experiments three saliva-VTM samples were processed through Norgen spin column kit and generated reproducible amplifications of the replicates $(n=2)$ with $\mathrm{Ct}$ values comparable to the reference $\mathrm{Ct}$ values. The outcome of this experiment cleared the way to determine $\mathrm{Ct}$ values of all the 24 positive and 31 negative reference saliva-VTM samples (Table 2(a), Table 2(b)). However, before applying the spin column purification method for RNA extraction from the 15 positive reference nasal swab samples, it was essential to individually characterise supernate and swab from the same sample tube. The objective behind this investigation was to determine 
Table 2. (a) Comparison of Ct values of microchip RT-PCR test with reference Ct of saliva-VTM positive samples; (b) Comparison of Ct values of microchip RT-PCR test with reference Ct of saliva-VTM negative samples.

(a)

\begin{tabular}{|c|c|c|c|c|c|c|}
\hline$\#$ & Sample ID & $\begin{array}{c}\text { Sample } \\
\text { Type }\end{array}$ & $\begin{array}{c}\text { Reference } \\
\mathrm{Ct}\end{array}$ & $\begin{array}{c}\text { Reference } \\
\text { Results } \\
\text { P/N }\end{array}$ & $\begin{array}{c}\text { Microchip } \\
\text { RT-PCR } \\
\text { Ct }\end{array}$ & $\begin{array}{c}\text { Microchip } \\
\text { RT-PCR } \\
\text { Results P/N }\end{array}$ \\
\hline 1 & 201210-079 & Saliva & 19.1 & Positive & 20.17 & Positive \\
\hline 2 & $201210-080$ & Saliva & 34.5 & Positive & 34.70 & Positive \\
\hline 3 & $201210-081$ & Saliva & 31.0 & Positive & 31.27 & Positive \\
\hline 4 & $201210-082$ & Saliva & 27.9 & Positive & 25.85 & Positive \\
\hline 5 & 201210-083 & Saliva & 31.9 & Positive & 28.21 & Positive \\
\hline 6 & 201210-084 & Saliva & 21.7 & Positive & 27.46 & Positive \\
\hline 7 & 201210-085 & Saliva & 19.9 & Positive & 20.14 & Positive \\
\hline 8 & $201210-086$ & Saliva & 26.9 & Positive & 27.01 & Positive \\
\hline 9 & 201210-087 & Saliva & 31.4 & Positive & 20.99 & Positive \\
\hline 10 & $201210-088$ & Saliva & 23.1 & Positive & 23.32 & Positive \\
\hline 11 & $201210-089$ & Saliva & 21.6 & Positive & 14.03 & Positive \\
\hline 12 & $201210-090$ & Saliva & 29.1 & Positive & 27.45 & Positive \\
\hline 13 & 201210-091 & Saliva & 28.3 & Positive & 27.90 & Positive \\
\hline 14 & $210122-113$ & Saliva & 25.55 & Positive & 23.75 & Positive \\
\hline 15 & $210122-114$ & Saliva & 25.56 & Positive & 27.43 & Positive \\
\hline 16 & $210122-115$ & Saliva & 24.53 & Positive & 23.37 & Positive \\
\hline 17 & $210122-116$ & Saliva & 25.05 & Positive & 28.26 & Positive \\
\hline 18 & $210122-117$ & Saliva & 30.66 & Positive & 26.60 & Positive \\
\hline 19 & $210122-118$ & Saliva & 31.12 & Positive & 29.62 & Positive \\
\hline 20 & 210122-119 & Saliva & 30.82 & Positive & 37.13 & Positive \\
\hline 21 & $210122-120$ & Saliva & 22.48 & Positive & 36.82 & Positive \\
\hline 22 & $210122-121$ & Saliva & 24.24 & Positive & 25.13 & Positive \\
\hline 23 & $210122-122$ & Saliva & 16.30 & Positive & 17.66 & Positive \\
\hline 24 & $210122-123$ & Saliva & 30.55 & Positive & 29.70 & Positive \\
\hline
\end{tabular}

(b)

\begin{tabular}{ccccccc}
\hline \# & Sample ID & $\begin{array}{c}\text { Sample } \\
\text { Type }\end{array}$ & $\begin{array}{c}\text { Reference } \\
\text { Ct }\end{array}$ & $\begin{array}{c}\text { Reference } \\
\text { Results } \\
\text { P/N }\end{array}$ & $\begin{array}{c}\text { Microchip } \\
\text { RT-PCR } \\
\text { Ct }\end{array}$ & $\begin{array}{c}\text { Microchip } \\
\text { RT-PCR } \\
\text { Results P/N }\end{array}$ \\
\hline 1 & $201210-045$ & Saliva & 0 & Negative & 0 & Negative \\
2 & $201210-046$ & Saliva & 0 & Negative & 0 & Negative \\
3 & $201210-047$ & Saliva & 0 & Negative & 0 & Negative \\
4 & $201210-048$ & Saliva & 0 & Negative & 0 & Negative \\
5 & $201210-049$ & Saliva & 0 & Negative & 0 & Negative \\
\hline
\end{tabular}




\section{Continued}

\begin{tabular}{|c|c|c|c|c|c|c|}
\hline 6 & 201210-050 & Saliva & 0 & Negative & 0 & Negative \\
\hline 7 & 201210-051 & Saliva & 0 & Negative & 0 & Negative \\
\hline 8 & 201210-052 & Saliva & 0 & Negative & 0 & Negative \\
\hline 9 & 201210-053 & Saliva & 0 & Negative & 0 & Negative \\
\hline 10 & $201210-054$ & Saliva & 0 & Negative & 0 & Negative \\
\hline 11 & 201210-055 & Saliva & 0 & Negative & 0 & Negative \\
\hline 12 & 201210-056 & Saliva & 0 & Negative & 0 & Negative \\
\hline 13 & 201210-057 & Saliva & 0 & Negative & 0 & Negative \\
\hline 14 & 201210-058 & Saliva & 0 & Negative & 0 & Negative \\
\hline 15 & 201210-059 & Saliva & 0 & Negative & 0 & Negative \\
\hline 16 & 201210-060 & Saliva & 0 & Negative & 0 & Negative \\
\hline 17 & $201210-061$ & Saliva & 0 & Negative & 0 & Negative \\
\hline 18 & 201210-062 & Saliva & 0 & Negative & 0 & Negative \\
\hline 19 & 201210-063 & Saliva & 0 & Negative & 0 & Negative \\
\hline 20 & 201210-064 & Saliva & 0 & Negative & 0 & Negative \\
\hline 21 & 201210-065 & Saliva & 0 & Negative & 0 & Negative \\
\hline 22 & 201210-066 & Saliva & 0 & Negative & 0 & Negative \\
\hline 23 & 201210-067 & Saliva & 0 & Negative & 0 & Negative \\
\hline 24 & 201210-068 & Saliva & 0 & Negative & 0 & Negative \\
\hline 25 & 201210-069 & Saliva & 0 & Negative & 0 & Negative \\
\hline 26 & 201210-070 & Saliva & 0 & Negative & 0 & Negative \\
\hline 27 & 201210-071 & Saliva & 0 & Negative & 0 & Negative \\
\hline 28 & 201210-072 & Saliva & 0 & Negative & 0 & Negative \\
\hline 29 & 201210-073 & Saliva & 0 & Negative & 0 & Negative \\
\hline 30 & 201210-074 & Saliva & 0 & Negative & 0 & Negative \\
\hline 31 & 201210-075 & Saliva & 0 & Negative & 0 & Negative \\
\hline
\end{tabular}

if only supernate instead of the swab could be used for RNA extraction from adequately homogenized sample after vortexing the sample tube.

Three nasal swab samples were chosen with high (Ct 10.72), mid (Ct 21.5), and low (Ct 30.8) viral RNA concentrations to account for wider range of samples. The comparative Ct values obtained during microchip RT-PCR from the supernate sample were about 3 cycles lower than the $\mathrm{Ct}$ value from the swab consequently suspended in the RNA storage buffer (Table 3, Figure 3 ). The difference in $\mathrm{Ct}$ value of 3 cycles is equivalent to about 10-fold difference in RNA concentration. These observations suggested that the supernate from the nasal swab tube contains about $90 \%$ of the RNA in the original nasal swab sample, and thus supernate portion is preferred over the swab portion for simplified processing. The data also suggested that the $\mathrm{Ct}$ from the supernate matched the reference 
Table 3. Ct values of microchip RT-PCR test for supernate and swab portions of nasal swab samples.

\begin{tabular}{cccccccc}
\hline \multirow{2}{*}{$\begin{array}{c}\text { PCR } \\
\text { Target }\end{array}$} & Sample & \multicolumn{2}{c}{$\# 16$} & \multicolumn{2}{c}{$\# 20$} & \multicolumn{2}{c}{$\# 10$} \\
\cline { 3 - 8 } & & Reference Ct 10.72 & \multicolumn{2}{c}{ Reference Ct 21.5 } & \multicolumn{2}{c}{ Reference Ct 30.8 } \\
\cline { 3 - 8 } & Sep 1 & 10.91 & 13.77 & 20.33 & 23.22 & 23.34 & 32.60 \\
& Rep 2 & 10.83 & 13.65 & 20.51 & 27.96 & 29.17 & 32.15 \\
& Rep 3 & 10.90 & 13.81 & 20.31 & 22.89 & 28.60 & 32.73 \\
N1 & Mean & 10.88 & 13.74 & 20.38 & 24.69 & 27.04 & 32.49 \\
& SD & 0.04 & 0.07 & 0.09 & 2.32 & 2.62 & 0.25 \\
& CV\% & 0.33 & 0.49 & 0.44 & 9.38 & 9.71 & 0.76 \\
\hline \multirow{4}{*}{ Hs } & Rep 1 & - -ve & $-v e$ & 29.5 & 32.26 & 23.08 & 25.43 \\
& Rep 2 & - -ve & $-v e$ & 28.05 & $-v e$ & 22.96 & 25.26 \\
& Rep 3 & - -ve & $-v e$ & 27.66 & 29.24 & 22.80 & 26.09 \\
& Mean & & & 28.40 & 30.75 & 22.95 & 25.59 \\
& SD & & & 0.79 & 1.51 & 0.11 & 0.36 \\
& CV\% & & & 2.79 & 4.91 & 0.50 & 1.40 \\
\hline
\end{tabular}

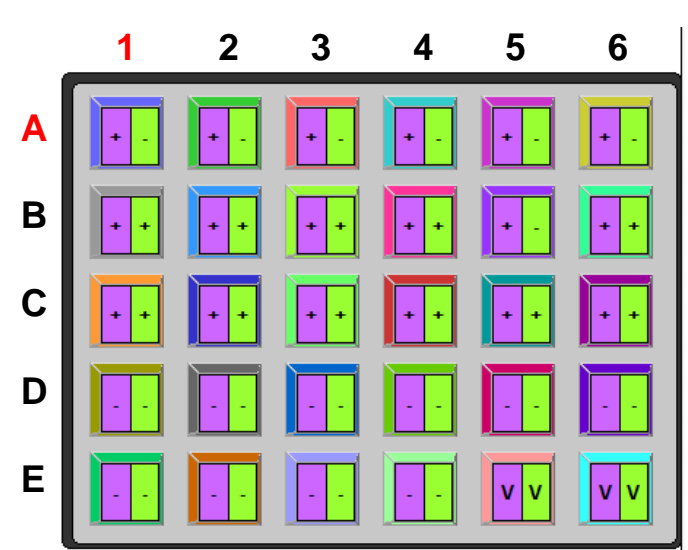

(a)

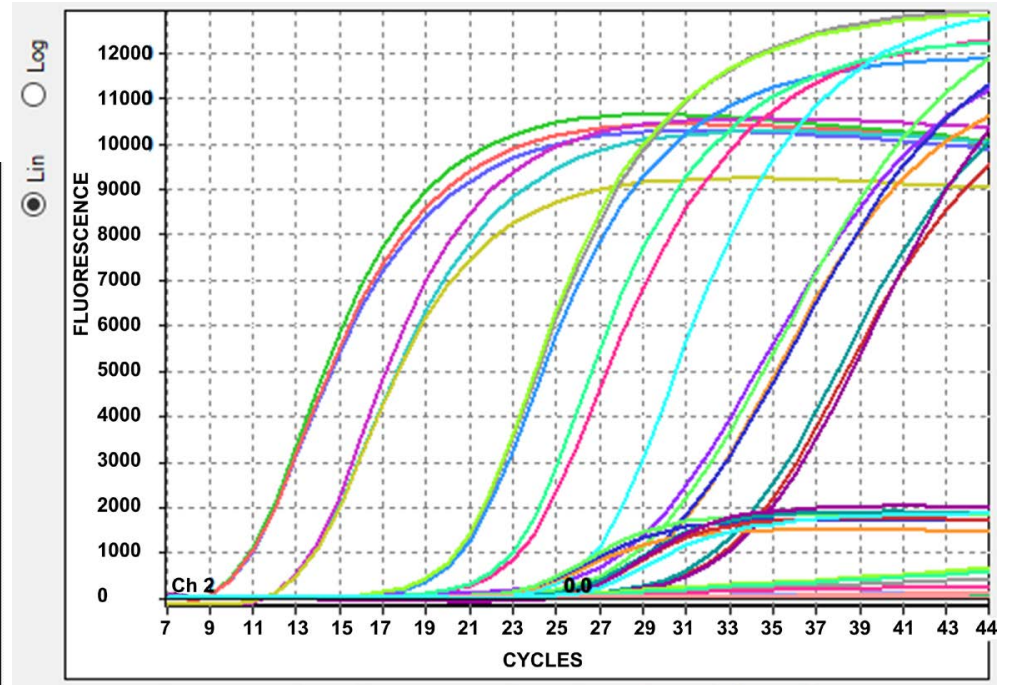

(b)

Figure 3. (a) Nasal swab supernate versus swab from the sample tube and N1 versus Hs competition $(\mathrm{n}=3)$ from 3 reference samples of high, mid and low reference Ct. The extraction replicates of extracted RNA from supernate of the sample tube (microwell A1-3) versus RNA A from the nasal swab of the same sample tube (microwell A 4 - 6). The Hs molecular target reports in the second and third sample but not from the high Ct sample. (b) Swab amplification curve behind the supernate amplification for each of the 3 samples.

Ct of the samples. This data indicated efficient dislodging of sample from the original swab into the VTM solution in the tube after homogenization by brief vortexing. Additionally, the data also displayed an interaction between the amplification/reporting of Hs molecular target used as IC to detect human speci- 
men in the samples and the number of copies of the SARS-CoV-2 reported by $\mathrm{N} 1$ with following correlation: the lower the $\mathrm{Ct}$ value of $\mathrm{N} 1$ target, the higher the reported Ct value of Hs target. To correctly assign sample results, a sample was considered positive if $\mathrm{N} 1$ target outcome was positive regardless of $\mathrm{Hs}$ target outcome (positive or negative). Using the supernate from all the 15 reference nasal samples, Ct values were determined using duplex microchip test system that matched the reference $\mathrm{Ct}$ values (Table 4).

Accuracy: The outcome of a diagnostic accuracy test is classified using the formula (1). In the studies described above, out of a total of 37 positive reference samples, 37 turned out to be positive and out of a total of 31 reference negative samples, 31 resulted in negative reflecting an accuracy of the test as 100\% (Table 5).

Specificity and sensitivity: in silico specificity of the primer-probe assays of the EUA molecular target panel was determined and presented by US-CDC

Table 4. Comparison of Ct values of Microchip RT-PCR test with reference Ct of nasal swab positive samples.

\begin{tabular}{|c|c|c|c|c|c|c|}
\hline$\#$ & Sample ID & Sample Type & $\begin{array}{c}\text { Reference } \\
\text { Ct }\end{array}$ & $\begin{array}{c}\text { Reference } \\
\text { Results } \\
\text { P/N }\end{array}$ & $\begin{array}{c}\text { Microchip } \\
\text { RT-PCR } \\
\text { Ct }\end{array}$ & $\begin{array}{c}\text { Microchip } \\
\text { RT-PCR } \\
\text { Results P/N }\end{array}$ \\
\hline 1 & $210122-126$ & Nasal swab & 16.42 & Positive & 21.20 & Positive \\
\hline 2 & $210122-127$ & Nasal swab & 23.52 & Positive & 21.99 & Positive \\
\hline 3 & $210122-129$ & Nasal swab & 26.26 & Positive & 24.52 & Positive \\
\hline 4 & $210122-130$ & Nasal swab & 25.93 & Positive & 21.25 & Positive \\
\hline 5 & $210122-131$ & Nasal swab & 15.17 & Positive & 16.93 & Positive \\
\hline 6 & $210122-132$ & Nasal swab & 12.29 & Positive & 10.45 & Positive \\
\hline 7 & $210122-134$ & Nasal swab & 30.80 & Positive & 29.00 & Positive \\
\hline 8 & $210122-135$ & Nasal swab & 15.53 & Positive & 12.79 & Positive \\
\hline 9 & $210122-136$ & Nasal swab & 28.12 & Positive & 30.68 & Positive \\
\hline 10 & $210122-140$ & Nasal swab & 10.72 & Positive & 10.90 & Positive \\
\hline 11 & $210122-141$ & Nasal swab & 15.09 & Positive & 13.26 & Positive \\
\hline 12 & $210122-142$ & Nasal swab & 18.17 & Positive & 14.69 & Positive \\
\hline 13 & $210122-143$ & Nasal swab & 14.74 & Positive & 11.01 & Positive \\
\hline 14 & $210122-144$ & Nasal swab & 21.53 & Positive & 20.40 & Positive \\
\hline 15 & $210122-146$ & Nasal swab & 20.27 & Positive & 21.08 & Positive \\
\hline
\end{tabular}

Table 5. Accuracy of microchip RT-PCR test system for saliva-VTM samples.

\begin{tabular}{ccc}
\hline Subjects & Number of samples & Accuracy of Microchip RT-PCR \\
\hline True positive samples & 39 & \\
True negative samples & 31 & $(39+31) / 70=100 \%$ \\
Total number of samples & 70 & \\
\hline
\end{tabular}


[https://www.fda.gov/media/134922/download]. To determine diagnostic specificity and diagnostic sensitivity, a set of reference clinical samples was analyzed using the duplex microchip test system.

In the present validation study, 31 reference negative saliva-VTM samples were tested as true negative, and the specificity was calculated as $100 \%$ using the formula (2) (Table 6). Accordingly, the testing of 24 reference positive saliva-VTM samples and 15 reference positive nasal swab samples (39 true positive samples) resulted in $100 \%$ sensitivity using the formula (3) (Table 6). These results are in line with Reich et al., 2021 where PCR with sensitivity of $94.7 \%$, specificity of $100 \%$, positive predictive value of $100 \%$ and a negative predictive value of $98.6 \%$ has been reported from COVID-19 patient nasopharyngeal samples compared with the composite reference standards in hospitalized patients [25].

Limit of Detection (LoD): The approximate LoD by duplex Microchip RT-PCR obtained in the study is tabulated in Table 7(a) where RNA concentration is shown in the spiked volume of $10 \mu \mathrm{l}$ per $100 \mu \mathrm{l}$ of the sample. It was determined using a 10-fold serial dilution of synthetic standard RNA spiked into negative

Table 6. Specificity and sensitivity of microchip RT-PCR test system.

\begin{tabular}{ccccccccc}
\hline & \multicolumn{8}{c}{ Microchip RT-PCR results } \\
\cline { 2 - 9 } Samples & Positive & Negative & $\begin{array}{c}\text { True Positive } \\
\text { TP }\end{array}$ & $\begin{array}{c}\text { True Negative } \\
\text { TN }\end{array}$ & $\begin{array}{c}\text { False Negative } \\
\text { FN }\end{array}$ & $\begin{array}{c}\text { False Positive } \\
\text { FP }\end{array}$ & $\begin{array}{c}\text { Specificity } \\
\%\end{array}$ & $\begin{array}{c}\text { Sensitivity } \\
\%\end{array}$ \\
\hline $\begin{array}{c}31^{*} \text { Negative } \\
24^{*}+15^{* *}(39) \\
\text { Positive }\end{array}$ & 0 & 31 & - & 31 & 0 & 0 & 100 & - \\
\hline
\end{tabular}

*saliva-VTM samples; ${ }^{* *}$ nasal swabs samples.

Table 7. LoD determination. (a) 10-fold serial dilution of the RNA standard; (b) 2-fold serial dilution of the RNA standard; (c) Confirmation of LoD.

(a)

\begin{tabular}{ccccc}
\hline & \multicolumn{2}{c}{ 2019-nCoV_N1 } & & \\
\hline $\begin{array}{c}\text { RNA Concentration in the spiked volume } \\
\text { (in copies) }\end{array}$ & $5 \times 10^{3}$ & $5 \times 10^{2}$ & $5 \times 10^{1}$ & $5 \times 10^{0}$ \\
\hline Positives/Total & $3 / 3$ & $3 / 3$ & $2 / 3$ & $3 / 3$ \\
Mean Ct & 24.80 & 28.36 & 32.08 & 36.31 \\
Standard Deviation (Ct) & 0.08 & 0.12 & 0.09 & 0.51 \\
\hline
\end{tabular}

(b)

\begin{tabular}{cccccccc}
\hline $\begin{array}{c}\text { RNA Concentration in the } \\
\text { spiked volume (in copies) }\end{array}$ & $2.0 \times 10^{2}$ & $1.0 \times 10^{2}$ & $5 \times 10^{1}$ & $2.5 \times 10^{1}$ & $1.25 \times 10^{1}$ & $6.25 \times 10^{0}$ & $3.12 \times 10^{0}$ \\
\hline Positives/Total & $3 / 3$ & $3 / 3$ & $3 / 3$ & $3 / 3$ & $3 / 3$ & $3 / 3$ & $1 / 3$ \\
Mean Ct & 28.76 & 29.95 & 31.12 & 33.36 & 38.58 & 35.59 & 39.38 \\
Standard Deviation (Ct) & 0.33 & 0.29 & 0.29 & 0.98 & 1.56 & 0.75 & NA \\
\hline
\end{tabular}


(c)

\begin{tabular}{ccccc}
\hline $\begin{array}{c}\text { RNA Concentration in spiked volume } \\
\text { (in copies) }\end{array}$ & $5 \times 10^{1}$ & $2.5 \times 10^{1}$ & $1.25 \times 10^{1}$ & $6.25 \times 10^{0}$ \\
\hline Positives/Total & $20 / 20$ & $20 / 20$ & $16 / 20$ & $9 / 20$ \\
Mean Ct & 33.40 & 33.78 & 35.45 & 38.31 \\
Standard Deviation (Ct) & 1.05 & 1.41 & 1.55 & 1.32 \\
\hline
\end{tabular}

saliva-VTM sample processed through spin column. The LoD determined by a 2 -fold serial dilution of the standard RNA was obtained to be in the range of $5 \times$ $10^{1}-3.12 \times 10^{0}$ copies $/ \mu$ in the spiked volume, as presented in Table 7(b). Determination of the positive agreement rates using 20 replicates $(n=20)$ from the 2 -fold serial dilution range resulted in positive agreement rates of $45 \%(9 / 20)$, $80 \%(16 / 20), 100 \%(20 / 20)$, and $100 \%(20 / 20)$, as shown in the Table $7(c)$. Thus, confirmed LoD was determined as a minimum concentration with positive agreement rate above $95 \%$ to be $2.5 \times 10^{1}$ copies $/ \mu$ in the spiked volume which corresponds to 2.5 copies/ $\mu \mathrm{l}$ in the original sample.

\section{Conclusion}

To successfully manage the infectious disease outbreaks, such as COVID-19, cost-effective, sensitive, and specific analysis is necessary. The speed, ease of use, and analytical performance of the validated microchip RT-PCR platform make it well suited for the screening of saliva-VTM and nasal swab samples. Reproducibility of results between RNA replicates of both reference clinical samples and genomic RNA standard was characterized across these validation experiments. A complete concordance among the data from these studies offering $100 \%$ specificity and sensitivity along with $100 \%$ accuracy supports this Microchip RT-PCR test system for Covid-19 detection as Research Use Only (RUO) or as a validated test subject to necessary validation in compliance with the local regulatory authority.

\section{Acknowledgements}

The support extended by Steven Hao in manufacturing of microchips is acknowledged.

\section{Conflicts of Interest}

The authors declare no conflicts of interest regarding the publication of this paper.

\section{References}

[1] Babiker, A., Myers, C.W., Hill, C.E. and Guarner, J. (2020) SARS-CoV-2 Testing. American Journal of Clinical Pathology, 153, 706-708.

https://doi.org/10.1093/ajcp/aqaa052

[2] Zhou, P., Yang, X.-L., Wang, X.-G., Hu, B., Zhang, L., Zhang, W., Si, H.-R., Zhu, Y., 
Li, B., Huang, C.-L., Chen, H.-D., Chen, J., Luo, Y., Guo, H., Jiang, R.-D., Liu, M.-Q., Chen, Y., She, X.-R., Wang, X., Zheng, X.-S., Zhao, K., Chen, Q.-J., Deng, F., Liu, L.-L., Yan, B., Zhan, F.-X., Wang, Y.-Y., Xiao, G.-F. and Shi, Z.-L. (2020) A Pneumonia Outbreak Associated with a New Coronavirus of Probable Bat Origin. Nature, 579, 270-273. https://doi.org/10.1038/s41586-020-2012-7

[3] Munne, M., Bhanothu, V., Bhor, V., Patel, V., Mahale, S.D. and Pande, S. (2021) Detection of SARS-CoV-2 Infection by RT-PCR Test: Factors Influencing Interpretation of Results. Virusdisease, 32, 1-3. https://doi.org/10.1007/s13337-021-00692-5

[4] Pearson, J.D., Trcka, D., Lu, Hyduk, S.J., Jen, M., Aynaud, M.-M., Hernández, J.J., Peidis, P., Barrios-Rodiles, M., Chan, K., Woodgett, J., Mazzulli, T., Attisano, L., Pelletier, L., Cybulsky, M.I., Wrana, J.L. and Bremner, R. (2021) Comparison of SARSCoV-2 Indirect and Direct RT-qPCR Detection Methods. Virology Journal, 18, Article No. 99. https://doi.org/10.1186/s12985-021-01574-4

[5] Razvan, C., Yaseen, I., Unrau, P.J., Lowe, C.F., Ritchie, G., Romney, M.G., Sin, D.D., Gill, S. and Slyadnev, M. (2012) Microchip RT-PCR Detection of Nasopharyngeal SARS-CoV-2 Samples. Journal of Molecular Diagnostics, 8, S1525-S1578.

[6] Gill, R., Gill, S., Gelimson, I., Slyadnev, M, Martinez, G., Gaines, M., Nunley, R. and Majoros, T. (2021) Color-Coding of Microchip RT-PCR Test System for SARS-CoV-2 Detection. Journal of Biosciences and Medicines, 9, 94-119. https://doi.org/10.4236/jbm.2021.95010

[7] Bogdanov, K.V., Nikulina, T.S., Lomaia, E.G., Slyadnev, M.N. and Zaritskey, A.Y. (2017) Identification of Oncogene Mutations in Leukemia Patients Using Microchip-Based PCR Analysis. Russian Journal of Bioorganic Chemistry, 43, 544-551. https://doi.org/10.1134/S1068162017040033

[8] Nikitin, M.M., Statsyuk, N.V., Frantsuzov, P.A., Dzhavakhiya, V.G. and Golikov, A.G. (2018) Matrix Approach to the Simultaneous Detection of Multiple Potato Pathogens by Real-Time PCR. Journal of Applied Microbiology, 124, 797-809. https://doi.org/10.1111/jam.13686

[9] Nikitin, M.M., Statsyuk, N.V., Frantsuzov, P.A., Pridannikov, M.V. and Golikov, A.G. (2017) Rapid and Simple Detection of Two Potato Cyst Nematode Species by Real-Time Multiplex PCR Using Preserved Microarray-Based Test Systems. Russian Journal of Nematology, 25, 51-60. http://www.russjnematology.com/Articles/rjn251/Nikitin_RJN2017 1 FIN2.pdf

[10] Gill, R., Gill, S., Slyadnev, M. and Stroganov, A. (2018) Identification and Quantitation of Cashmere (Pashmina) Fiber and Wool Using Novel Microchip Based Real-Time PCR Technology. Journal of Textile Science and Technology, 4, 141-150. https://doi.org/10.4236/jtst.2018.44010

[11] Tong, R., Zhang, L., Song, Q., Hu, C, Chen, X., Lou, K., Gong, X., Gao, Y. and Wen, W. (2019) A Fully Portable Microchip Real-Time Polymerase Chain Reaction for Rapid Detection of Pathogen. Electrophoresis, 40, 1699-1707. https://doi.org/10.1002/elps.201900090

[12] Abdulina, D.R., Iutynska, G.O., Anjskina, A.I. and Nikitin, M.M. (2020) Detection of Sulfate-Reducing Bacteria from Various Ecotopes by Real-Time PCR. Biotechnologia Acta, 13, 38-47. https://doi.org/10.15407/biotech13.02.038

[13] Wozniak, A., Cerda, A., Ibarra-Henríquez, C., Sebastian, V., Armijo, G., Lamig, L. Miranda, C., Lagos, M., Solari, S., Guzmán, A.M., Quiroga, M., Hitschfeld, M., Riveras, E., Ferrés, M., Gutiérrez, R.A. and García, P. (2020) A Simple RNA Preparation Method for SARS-CoV-2 Detection by RT-qPCR. Scientific Reports, 10, Article No. 16608. https://doi.org/10.1038/s41598-020-73616-w

[14] Graham, T.G.W., Dugast-Darzacq, C., Dailey, G.M., Nguyenla, X.H., Van Dis, E.V., 
Esbin, M.N., Abidi, A., Stanley, S.A., Darzacq, X. and Tjian, R. (2021) Open-Source RNA Extraction and RT-qPCR Methods for SARS-CoV-2 Detection. PLoS ONE, 16, e0246647. https://doi.org/10.1371/journal.pone.0246647

[15] Bruce, E.A., Huang, M.L., Perchetti, G.A. and Botten, J.W. (2021) Direct RT-qPCR Detection of SARS-CoV-2 RNA from Patient Nasopharyngeal Swabs without an RNA Extraction Step. https://doi.org/10.1101/2020.03.20.001008

[16] Kirkland, P.D. and Frost, M.J. (2020) The Impact of Viral Transport Media on PCR Assay Results for the Detection of Nucleic Acid from SARS-CoV-2. Pathology, 52, 811-814. https://doi.org/10.1016/j.pathol.2020.09.013

[17] Banada, P., Elson, D., Daivaa, N., Park, C., Desind, C., Montalvan, L., Kwiatkowski, R., Chakravorty, S., Alland, D. and Xie, Y.L. (2021) Evaluation of Sample Collection and Transport Strategies to Enhance Yield, Accessibility, and Biosafety of COVID-19 RT-PCR Testing. https://doi.org/10.1101/2021.03.03.21251172

[18] Coetzee, J.F. (2004) Evaluating Diagnostic Tests. Southern African Journal of Anaesthesia \& Analgesia, 10, 7-16. https://doi.org/10.1080/22201173.2004.10872376

[19] Bezier, C., Anthoine, G. and Charki, A. (2020) Reliability of Real-Time RT-PCR Tests to Detect SARS-Cov-2: A Literature Review. International Journal of Metrology and Quality Engineering, 11, Article No. 13. https://doi.org/10.1051/ijmqe/2020014

[20] Vogels, C.B.F., Watkins, A.E., Harden, C.A., Brackney, D.E., Shafer, J., Wang, J., Caraballo, C., Kalinich, C.C., Ott, I.M., Fauver, J.R., Kudo, E., Lu, P., Venkataraman, A., Tokuyama, M., Moore, A.J., Muenker, M.C., Casanovas-Massana, A., Fournier, J. and Grubaugh, N.D. (2021) SalivaDirect: A Simplified and Flexible Platform to Enhance SARSCoV-2 Testing Capacity. MedRxiv, 2, 263-280. https://doi.org/10.1101/2020.08.03.20167791

[21] Wei, S., Kohl, E., Djandji, A., Morgan, S., Whittier, S., Mansukhani, M., Hod, E., D’Alton, M., Suh, Y. and Williams, S. (2020) Direct Diagnostic Testing of SARS-CoV-2 without the Need for Prior RNA Extraction. Science Reports, 11, Article No. 2402. https://doi.org/10.1038/s41598-021-81487-y

[22] Poggiali, E., Vercelli, A., Vadacca, G.B., Schiavo, R., Mazzoni, G., Loannilli, E., Demichele, E. and Magnacavallo, A. (2020) Negative Nasopharyngeal Swabs in COVID-19 Pneumonia: The Experience of an Italian Emergency Department (Piacenza) during the First Month of the Italian Epidemic. Acta BioMedica, 91, e2020024.

[23] Pan, Y., Long, L., Zhang, D., Yuan, T., Cui, S., Yang, P. and Ren, S. (2020) Potential False-Negative Nucleic Acid Testing Results for Severe Acute Respiratory Syndrome Coronavirus 2 from Thermal Inactivation of Samples with Low Viral Loads. Clinical Chemistry, 66, 794-801. https://doi.org/10.1093/clinchem/hvaa091

[24] Jarvis, K.F. and Kelley, J.B. (2021) Temporal Dynamics of Viral Load and False Negative Rate Influence the Levels of Testing Necessary to Combat COVID-19 Spread. Scientific Reports, 11, Article No. 9221. https://doi.org/10.1038/s41598-021-88498-9

[25] Reich, N., Lowe, C.F., Puddicombe, D., Matic, N., Greiner, J., Simons, J., Leung, V., Chu, T., Naik, H., Myles, N., Burns, L., Romney, M.G., Ritchie, G., Champagne, S., Dooley, K., Sekirov, J. and Stefanovic, A. (2021) Diagnostic Accuracy of RT-PCR for Detection of SARS-CoV-2 Compared to a "Composite Reference Standard" in Hospitalized Patients. MedRxiv. https://doi.org/10.1101/2021.02.18.21252016 